\title{
Addressing the Need for Alternative Transportation Fuels: The Joint BioEnergy Institute
}

Harvey W. Blanch ${ }^{\dagger, \hbar, \S}$, Paul D. Adams ${ }^{\dagger, \S, \pi}$, Katherine M. Andrews-Cramer ${ }^{\dagger, \iota}$, Wolf B. Frommer ${ }^{\dagger, \S, * *}$, Blake A.

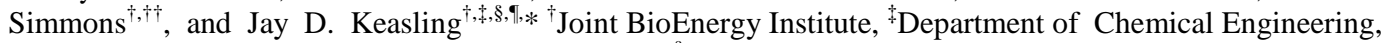
University of California, Berkeley California 94720, ${ }^{\S}$ Physical Biosciences Division, Lawrence Berkeley National Laboratory, Berkeley, California 94720, ${ }^{\mathrm{T}}$ Department of Bioengineering, University of California, Berkeley, California 94720, 'Sandia National Laboratories, Albuquerque, New Mexico 87185, **Department of Plant Biology, Carnegie Institute for Science, Stanford, California 94305, and ${ }^{\dagger \dagger}$ Sandia National Laboratories, Livermore, California 94551

Today, carbon-rich fossil fuels, primarily oil, coal, and natural gas, provide $85 \%$ of the energy consumed in the U.S. As world demand increases, oil reserves may become rapidly depleted (1). Fossil fuel use increases $\mathrm{CO}_{2}$ emissions and raises the risk of global warming. The high energy content of liquid hydrocarbon fuels makes them the preferred energy source for all modes of transportation. In the U.S. alone, transportation consumes $>13.8$ million barrels of oil per day and generates "0.5 gigatons of carbon per year (2). This release of greenhouse gases has spurred research into alternative, nonfossil energy sources. Among the options (nuclear, concentrated solar thermal, geothermal, hydroelectric, wind, solar, and biomass), only biomass has the potential to provide a high-energy-content transportation fuel. Biomass is a renewable resource that can be converted into carbon-neutral transporation fuels.

Currently, biofuels such as ethanol are produced largely from grains, but there is a large, untapped resource (estimated at more than a billion tons per year) of plant biomass that could be utilized as a renewable, domestic source of liquid fuels. Well-established processes convert the starch content of the grain into sugars that can be fermented to ethanol. The energy efficiency of starch-based biofuels is however not optimal, while plant cell walls (lignocellulose) represent a huge untapped source of energy (3). Plantderived biomass contains cellulose, which is more difficult to convert to sugars; hemicellulose, which contains a diversity of carbohydrates that have to be efficiently degraded by microorganisms to fuels; and lignin, which is recalcitrant to degradation and prevents cost-effective fermentation. The development of cost-effective and energy-efficient processes to transform lignocellulosic biomass into fuels is hampered by significant roadblocks, including the lack of specifically developed energy crops, the difficulty in separating biomass components, low activity of enzymes used to deconstruct biomass, and the inhibitory effect of fuels and processing byproducts on organisms responsible for producing fuels from biomass monomers.

The Joint BioEnergy Institute (JBEI) is a U.S. Department of Energy (DOE) Bioenergy Research Center that will address these roadblocks in biofuels production. JBEI draws on the expertise and capabilities of three national laboratories (Lawrence Berkeley National Laboratory (LBNL), Sandia National Laboratories (SNL), and Lawrence Livermore National Laboratory (LLNL)), two leading U.S. universities (University of California campuses at Berkeley (UCB) and Davis (UCD)), and a foundation (Carnegie Institute for Science, Stanford) to develop the scientific and technological base needed to convert the energy stored in lignocellulose into transportation fuels and commodity chemicals. Established scientists from the participating organizations are leading teams of researchers to solve the key scientific problems and develop the tools and infrastructure that will enable other researchers and companies to rapidly develop new biofuels and scale production to meet U.S. transportation needs and to develop and rapidly transition new technologies to the commercial sector.

JBEI's biomass-to-biofuels research approach is based in three interrelated scientific divisions and a technologies division. The Feedstocks Division will develop improved plant energy crops to serve as the raw materials for biofuels. The Deconstruction Division will investigate the conversion of this lignocellulosic plant material to sugar and aromatics. The Fuels Synthesis Division will create microbes that can efficiently convert sugar and aromatics into ethanol and other biofuels. JBEI's cross-cutting Technologies Division will develop and optimize a set of enabling technologies including high-throughput, chipbased, and 'omics platforms; tools for synthetic biology; multi-scale imaging facilities; and integrated data analysis to support and integrate JBEI's scientific program. 


\section{Systems Biology}

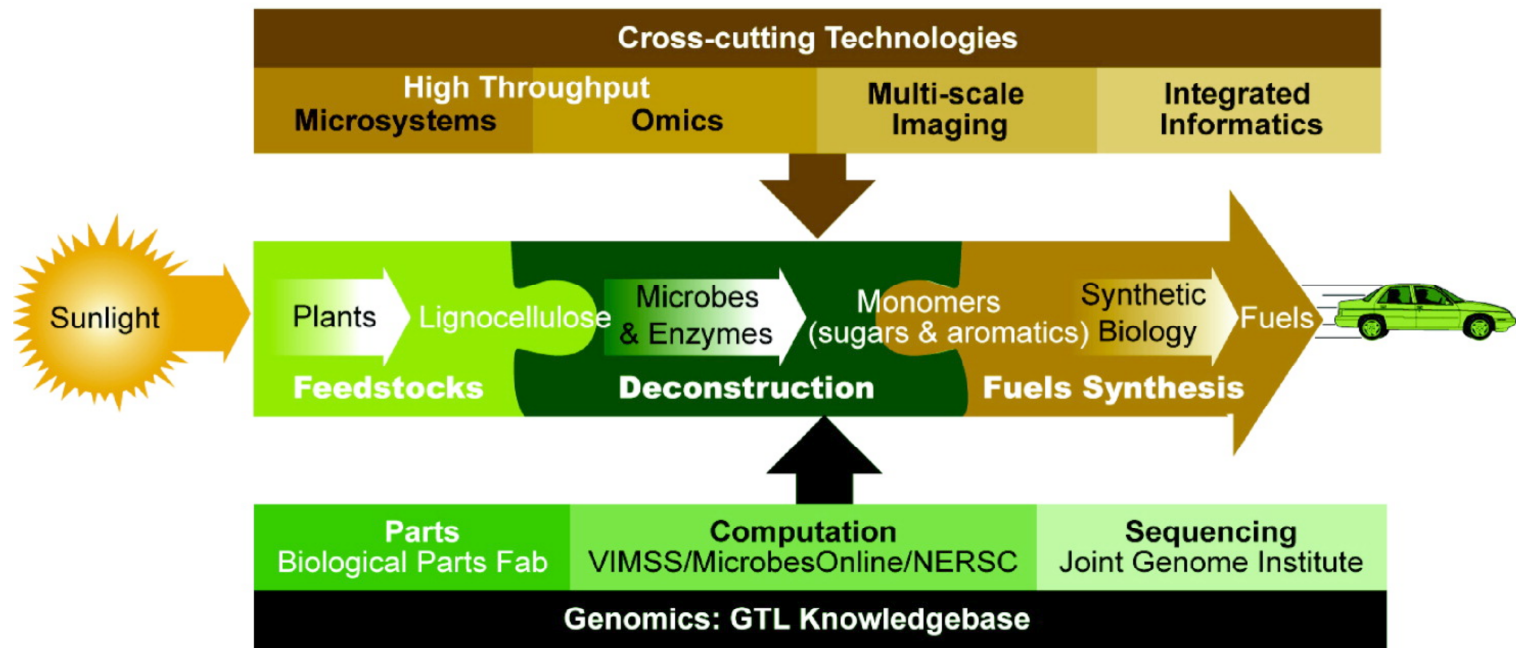

Figure 1. Overview of JBEI structure and research pipeline, courtesy of LBNL Creative Services Office.

\section{Energy Feedstocks: Understanding Recalcitrant Biomass.}

Atmospheric carbon dioxide is fixed by plants into carbohydrates through photosynthesis. Sugar cane and beet roots store large amounts of simple sugars, corn grain and wheat kernels store carbon as starch, and cellulose and hemicellulose are found in the leaves of all agricultural crops, as well as in the leaves and wood of trees. Most ethanol for fuel use today is produced from corn grain, and the technology for breaking down starch into simple sugars is well developed. Lignocellulosic biomass, such as wood, forest product residues, grasses, agricultural residues, and specialty energy crops, can provide much larger amounts of biomass for production of transportation fuels. However, lignocellulosic biomass is resistant to breakdown; plants have evolved complex means to employ cellulose and hemicellulose as structural materials that are very resistant to microbial attack. Lignin, a polyphenolic material, serves to strengthen the cellulosic material to form the plant cell wall, which provides resistance to pests and pathogens. The crystalline cellulose core of cell walls is very resistant to chemical and biological breakdown, and the complex structures of the cell wall also contribute to its recalcitrance. Research in JBEI's Feedstocks Division is directed at overcoming the recalcitrance of lignocellulosic plant matter so that it can be more easily deconstructed.

The main objectives of the Feedstocks Division are to elucidate the mechanisms involved in synthesis of plant cell wall constituents using high-throughput functional genomics and glycomics and to expand knowledge of lignin polymerization to allow the development of plants with novel types of lignin with equivalent biological function but with improved susceptibility to enzymatic and chemical depolymerization.

\section{Deconstruction: Converting Lignocellulosic Biomass to Sugars.}

The most direct approach to overcoming the recalcitrance of biomass relies on pretreatment by mechanical or chemical methods. Pretreatment aims to decrease the crystallinity of cellulose and increase the accessibility of the bio-mass for subsequent hydrolysis. Biomass pretreatment by dilute acid hydrolyzes the hemicellulose component, whereas treatment with alkali removes part of the lignin. These approaches, however, are not economically optimal. Other pretreatment approaches include steam and alkaline explosive decompression and hydrothermolysis. All of these pretreatment methods expose the cellulose fibers and make them more accessible to cellulase enzymes, which can then hydrolyze the cellulose to fermentable sugars. Enzymatic hydrolysis does not produce byproducts and thus offers the possibility of improving the costs of biofuels production.

Cellulolytic microorganisms (fungi and bacteria) produce enzymes that act synergistically to hydrolyze plant cell wall materials. Presently, our understanding of the fundamental mechanisms of enzymatic cellulose degradation is limited. Fungi produce three types of cellulolytic enzymes. Random-acting 
endoglucanases produce free ends from cellulose fibrils that can be degraded by exoglucanases, which produce the glucose dimer cellobiose. The third type of enzyme, \$-glucosidase, hydrolyzes the released cellobiose to produce glucose. Some bacteria employ a molecular "machine", the cellulosome, to break down cellulose.

Hemicellulose is degraded by a class of enzymes known as hemicellulases, which are multidomain enzymes containing structurally discrete catalytic and noncatalytic domains. Hemicellulases from different organisms are classified generally as either glycoside hydrolases or carbohydrate esterases, which hydrolyze acetate or ferulic acid side groups of the hemicellulose polymer, respectively. Compared to the research and development effort in the scientific community to understand and optimize cellulase enzymes, very little is known about the exact mechanisms of hemicellulases, and methods to engineer these enzymes are nascent. Lignin, the third major component of biomass, is the component most resistant to enzymatic attack. Lignases generally consist of a family of enzymes including phenol oxidase (laccase), peroxidases (lignin peroxidase), and manganese peroxidase.

Thus, the major objectives of JBEI's Deconstruction Division are i) to improve pretreatment methods with broad applicability to a range of feedstocks, ii) to explore new sources of lignocellulolytic enzymes from natural environments, relying on high-throughput protein production and directed evolution using on-chip technologies, iii) to examine microbial communities for new sources of cellulolytic and lignolytic enzymes, and iv) to develop lignin models and lignase assays that enable the creation of modified ligninases for enhanced degradation and conversion of modified lignin.

\section{Fuel Synthesis: Capturing the Energy Content of Sugars.}

Sugars derived from starch-based biomass such as corn are readily fermented to ethanol, because they are present in nearly pure solutions. In contrast, lignocellulose deconstruction results in both five- and six-carbon sugars together with a number of inhibitory compounds, including organic acids, furan derivatives, phenolics, and inorganics. Hardwoods and agricultural residues contain 5-25\% pentose sugars, primarily xylose and arabinose.

These are not fermented to ethanol by the most commonly used yeast, Saccharomyces cerevisiae. Anaerobic bacteria ferment pentose sugars but are typically inhibited by low concentrations of ethanol and other byproducts from deconstruction. Filamentous fungi are able to tolerate these inhibitors but grow and produce ethanol too slowly to be commercially attractive.

The challenge in the biofuels production division of JBEI is thus to convert all of the monomer sugars (hexoses and pentoses) released from depolymerization of lignocellulosic biomass into transportation fuels and other chemicals. Accomplishing this objective will first rely on developing and improving fuel production systems in selected model microorganisms: the bacterium Escherichia coli, the yeast $S$. cerevisiae, and the thermoacidophilic archaeon Sulfolobus solfataricus. JBEI is initially employing E. coli and S. cerevisiae strains that have been previously engineered to produce ethanol from five- and six-carbon sugars. All three hosts will be engineered to improve their tolerance to byproducts formed during biomass processing and to high concentrations of ethanol and other fuel products.

JBEI will develop biochemical synthesis pathways for production of a range of other candidate fuel molecules and chemicals that are currently based on petroleum feedstocks. We will construct and validate these pathways in E. coli and then, when functional, introduce them into S. cerevisiae and S. solfataricus. JBEI is initially targeting five existing or proposed fuel molecules: ethanol, butanol, isopentanol, hexadecane, and geranyl decanoate ester. Unlike ethanol, these potential fuel molecules may be used to power jet and diesel engines and can be distributed via existing infrastructure that is used to distribute petroleum-based fuels.

\section{Technologies for Biofuels Research and Production.}

Successfully meeting JBEI goals is critically dependent on the application and development of advanced technologies deployed in the context of a research environment that is fully integrated through state-of-the-art information systems and informatics methods. The JBEI Technologies Division will develop and implement technologies that can be applied to the research in each of the scientific and engineering divisions. Using high-throughput protein expression, purification, and screening, JBEI researchers will generate thousands of gene clones per year, fully characterize the plant cell wall synthesis machinery by synthesis and tagging to identify functional complexes, and perform functional analysis of tens of thousands of wild-type and engineered lignocellulose-degrading enzymes.

In functional genomics, JBEI researchers will characterize the transcript and protein profiles of natural and engineered organisms including plants, undertake metabolite and flux profiling of modified 
organisms to optimize fuel production, and perform high-throughput glycomics. In the area of synthetic biology, JBEI will develop platform hosts for the production of enzymes and fuels and create parts and devices for the construction of new fuel-generating organisms and improved plants. Finally, in highthroughput imaging, JBEI researchers will develop new and improved technologies for visualizing cell walls. The technologies developed within JBEI will be of general use for a wide variety of biological applications and will benefit the biofuels research com-munity, GTL, and other DOE initiatives.

\section{A Single Facility Integrates JBEI's Research.}

JBEI is designed to be a dynamic organization with all research teams working together at a single location. This colocation of researchers will enable scientists to share their ideas, develop technologies that will benefit all scientific divisions, and ad-dress cellulosic biomass problems at a systems level. JBEI is positioned to take advantage of the significant capabilities of its partners and other institutions and companies in the San Francisco Bay Area. JBEI's close working relationships with its industry partners will ensure that JBEI creates the fundamental knowledge and scalable technologies to solve real-world problems in commercial-scale biofuels production.

\section{Conclusions and Perspectives.}

The challenges in converting lignocellulosic feedstocks into transportation fuels are significant. The integrated approach taken by JBEI's researchers will realize basic science and engineering developments to meet these challenges. The potential payoffs from this research are significant: renewable, carbon-neutral transportation fuels; lessening the impact of global warming; and reducing our reliance on foreign oil while improving trade balances.

\section{REFERENCES}

1. National Research Council (2006) Trends in Oil Sup-ply and Demand, the Potential for Peaking of Conventional Oil Production and Possible Mitigation Op-tions, National Academies Press, Washington, DC.

2. U.S. Department of Energy (2005) Emission of Green-house Gases in the United States, www.eia.doe.gov/ oiaf/1605/flash/flashkbcb.html.

3. U.S. Department of Energy and U.S. Department of Ag-riculture (2005) Biomass as Feedstock for a Bioenergy and Bioproducts Industry: The Technical Feasibil-ity of a Billion-Ton Annual Supply, www.osti.gov/ bridge.

This work was supported by the Director, Office of Science, Office of Basic Energy Sciences, of the U.S. Department of Energy under Contract No. DE-AC02-05CH11231.

This work was supported by the Assistant Secretary for Energy Efficiency and Renewable Energy, Office of Building Technology, State, and Community Programs, of the U.S. Department of Energy under Contract No. DE-AC02-05CH11231. 